\title{
Unilateral Posterior Crossbite - Shoot It At Sight. A Review
}

\author{
Dr.Narmadha Sudhakar ${ }^{1}$ Dr.Saravana Dinesh ${ }^{2}$ \\ Saveetha Dental College
}

\begin{abstract}
This review is undertaken to see the effects of early orthodontic management of unilateral posterior crossbite (UPC). UPC is often accompanied by lateral mandibular shift and mandibular deviation because of the reduction in the width of the maxillary dental arch. Correction of early orthodontic treatment of UPC is more complex than it appears. Treatment success is high if it is started early. To develop an appropriate treatment plan, it is first necessary to determine if there is a functional jaw shift on closing, it is dental, skeletal or both or it is related only to maxilla or both the jaws. Evidence that crossbite are not self-correcting and have some association with TMJ disorders. It can be difficult to treat UCB in adults without a combination of orthodontics and surgery. Treatment of UCB generally involves symmetric expansion of the maxillary arch, removal of selective occlusal interferences and elimination of the mandibular functional shift. The general practitioner or dentist must be able to diagnose UPC successfully and provide treatment or referral to take advantage of the benefits of early treatment.
\end{abstract}

Key Words: cross bite ; early treatment ; malocclusion;appliances

\section{Introduction:}

Posterior crossbite is one of the most prevalent malocclusions in the primary and early mixed dentition. It is reported to occur between $8 \%$ and $22 \%{ }^{(1)}$. It is defined as any abnormal buccal-lingual relation between opposing molars, premolars or both in centric occlusion. The most common form of posterior crossbite is a unilateral presentation with a functional shift of the mandible towards the crossbite side ${ }^{(2)}$. A posterior crossbite is believed to be transferred from primary to permanent dentition and can have long-term effects on the growth of jaws ${ }^{(1)}$. A unilateral posterior crossbite is usually due to a bilaterally underdeveloped maxilla and a shifting of the mandible to one side during closure. Leighton (1966) reported that unilateral posterior crossbites first appear between 19 months and 5 years of age ${ }^{(3)}$. Studies of adolescents and adults have revealed that patients with posterior crossbite have an increased risk to develop cranio-mandibular disorders showing more signs and symptoms of these problems. Therefore early treatment is often advised to normalize the occlusion and create conditions for normal occlusal development ${ }^{(1)}$.

\section{Etiology:}

Crossbite has been attributed to skeletal, muscular or dental factors. Evidence indicates that causative factors can be related to genetic, congenital, environmental, functional or from oral habits (digit sucking). A small maxilla to mandible width ratio may arise from genetic or environmental factors. Upper airway obstruction in the form of hypertrophied adenoids or tonsils and allergic rhinitis can result in mouth breathing and also who have been intubated during infancy are correlated with the development of posterior crossbites ${ }^{(2)}$. An insufficient maxillary arch width typically results in a unilateral posterior crossbite with an associated lateral mandibular displacement which is also known as mandibular deviation or shift. A unilateral posterior crossbite usually presents with a mandibular midline discrepancy. When the maxilla is severely constricted, a bilateral posterior crossbite will present clinically ${ }^{(4)}$. In a review of the literature by Allen et al, the potential etiologic factors for posterior crossbite were given as follows- prolonged retention and premature loss of deciduous teeth, crowding, palatal cleft, genetic control, arch deficiencies, abnormalities in tooth anatomy or eruption sequence, oral digit habits, oral respiration during critical growth periods and malfunctioning TMJ's ${ }^{(5)}$.

It is well established that children with a unilateral posterior crossbite exhibit unusual chewing patterns when chewing on the affected side and this is characterized by an increased frequency of reverse sequencing (Lewin 1985).Usually the mandible deviates laterally towards the bolus side and then during closure, medially, through the trans-cuspal and intercuspal phases of mastication. In Reverse sequencing the mandible first deviates medially and then laterally, thus ensuring overlap of opposing dental occlusal surfaces. This reverse chewing pattern is dependent on central motor control. Reverse- sequencing chewing cycles occur on the crossbite side only. This is why a unilateral posterior crossbite is characterized by both dental and functional asymmetry. Such asymmetries may be prevented by orthodontic therapy at an early stage in development ${ }^{(6)}$.

\section{CLASSIFICATION:}

Though there are many classifications, the most widely used one is given by Lorente in 2002: 


\begin{tabular}{|c|c|c|}
\hline \multirow{3}{*}{ UCB-TRANSVERSE } & \multirow{3}{*}{ ANAMOLIES } & UCB with normal maxilla and constricted dento-alveolar process \\
\hline & & UCB with normal maxilla and asymmetrically-constricted dento-alveolar process \\
\hline & & UCB with constricted maxilla \\
\hline
\end{tabular}

\section{RATIONALE OF EARLY TREATMENT:}

Posterior crossbites that result from functional shift of the mandible should be treated as soon as clinically feasible after they are found. Such a shift in the posture of the mandible from its first contact in a centric posture to maximum intercuspation may result in a number of sequella in both hard and soft tissue. The changes that may occur include,

$>$ Compensatory changes in the TMJ articulation

$>$ The development of skeletal asymmetries

$>$ Modifications of soft tissue growth and

$>$ Attrition of the primary and secondary teeth Indications for palatal expansion:

- Relief of posterior crossbite with skeletal component.

- Gaining a small amount of space to relieve anterior crowding.

The secondary effect of palatal expansion is the direct result of increasing the transverse direction. This results in the total arch circumference increase that is approximately $75 \%$ of increase in width. Early correction of posterior crossbites may help prevent the signs and symptoms of TMJ disorders ${ }^{(7)}$.

\section{DIAGNOSIS:}

History- During the dental history collection, it must be determined if the patient had a digit-sucking problem or mouth breathing problem, and if so, its frequency, intensity, duration and persistence. In addition to the frequently observed proclination of the maxillary incisors with the creation of an anterior open bite, strong and persistent sucking habits can cause a narrowing of the maxillary dental arch in the transverse dimension as well as compensatory lingual tipping of the mandibular buccal segments. Such narrowing may or may not create an apparent dental crossbite ${ }^{(7)}$.

Clinical examination--Bilateral clicks can be observed in the TMJ with mandibular deviation on closing and opening of the mouth ${ }^{(11)}$. The patient is asked to open his/her mouth as wide as possible and keep it open for a short period of time. Possible mandible shifting is then evaluated by having the patient close the mandible slowly from maximum opening until the first contact of centric occlusion while the tongue is curled back toward the soft palate. The amount and direction of any mandible shifting between first contact and maximum intercuspation should be noted ${ }^{(7)}$.

Diagnostic records - The study casts is to be analysed and check for the relationships of the deciduous canines and second molars, crossbites and midline deviations should be recorded ${ }^{(5)}$. A panoramic radiographic is ideal. An occlusal radiograph is used to assess the mid-palatal suture patency. Radiographs also indicate formation stages of permanent teeth's developing roots and the resorption of their primary precursors also be checked for congenitally missing teeth ${ }^{(7)}$.

\section{TREATMENT TIMING:}

During the deciduous and early mixed dentition stages (under 8years) smaller forces can be used to achieve sutural expansion, as evidenced by a midline diastema during expansion or by radiographic images that shows opening of the suture ${ }^{(2)}$. Early treatment can prevent signs and symptoms of temperomandibular disorder and associated mandibular dysfunction and also facial asymmetry caused by posterior crossbites ${ }^{(13)}$. Another advantage of early treatment is improvement of maxillary arch length deficiency secondary to maxillary constriction because the permanent incisors are afforded more space before or during eruption than if the crossbite is treated at a later stage $e^{(2)}$. Treatment of functional crossbite by maxillary expansion is, therefore, best carried out during the late deciduous or early mixed dentition stages.

\section{Treatment And Its Methods:}

Treatment of functional posterior crossbite involves expansion of the maxillary arch, removal of occlusal interferences and elimination of the functional shift ${ }^{(2)}$. There are some differences in the techniques used to increase the transverse dimensions due to the midpalatal suture in the maxilla and its other sutural connections to the facial skeleton ${ }^{(7)}$. Maxillary expansion with the Quad-helix appliance is suggested as an effective treatment choice. And several other devices are available for the correction of functional posterior crossbites such as rapid palatal expanders, quad-helix appliances, slow maxillary expansion appliances such as nickel-titanium alloy devices, tandem loop, warsh and removable maxillary expansion devices ${ }^{(15)}$. Rapid maxillary expansion can be used in the deciduous, early mixed or early permanent dentition stages by using a Haas, hyrax or superscrew expander. Here some spontaneous increase occurs in the intercanine width of the mandibular permanent dentition. In patients with neuromuscularly controlled displacement of the mandible, a 
so-called reflex-releasing stabilizing bite splint can be employed as intercuspal position deviating from the structural position ${ }^{(16)}$.

\section{Appliances common to both arches:}

The type of device that is most frequently used in either arch is the acrylic-based removable appliance with one or more expansion screws. Typically the mandibular appliance is constructed with a single expansion screw located at the midline lingual to the central incisors. The maxillary type may have 1 or 2 screws, usually located transversely over the median raphe. The most commonly used clasps to engage the proximal buccal undercuts are Adams or ball clasps with C-clasps.

Changes in the transverse dimension are achieved by turning the screws between the appliances once or twice a week. As the screw is turned, the acrylic body of the appliance applies pressure to the teeth's lingual surfaces and alveolar process to achieve movement. Contact between the teeth and the appliance is only along the junction of acrylic and gingival margins. This creates a narrow band of contact and thus a tipping movement. Adding a bite plane to the appliance helps to achieve more bodily movement which increase the appliance's retention and reduced intercuspation of the opposing teeth, thus enhancing movement. Lip bumper can also be used in both the arches to achieve dentoalveolar expansion, but these are mostly used in the mandible ${ }^{(7)}$.

\section{Maxillary appliances:}

RPE: Rapid Palatal Expansion is the most effective with a fixed appliance with a Jackscrew device which is usually turned one time a day. The predominant effect in a growing child is the opening of the midpalatal suture ${ }^{(4)}$. The patient should be warned that a midline maxillary diastema will be created initially. During the retention stage, the diastema will gradually close by dental tipping as transeptal fibres approximate the central incisors ${ }^{(2)}$. When selecting an expansion method, the patient's age, skeletal and muscular pattern, degree of maxillary constriction, and habits should all be considered ${ }^{(4)}$. The Hass- and Hyrax-type rapid palatal expansion appliances are employed to achieve crossbites correction via separation of the midpalatal suture ${ }^{(7)}$

AMEX: Asymmetric Maxillary Expansion -This appliance used for the treatment of true unilateral crossbite, the aim should be move selected teeth on the constricted side of the maxillary arch. If conventional expansion appliances are preferred to treat true unilateral crossbite, then the maxillary dental arch will be expanded bilaterally resulting in undesirable overexpansion of the unaffected side ${ }^{(9)}$. In this situation, additional appointments are required for compensatory orthodontic tooth movements on both non crossbite and crossbite sides. A simple way is to treat a true unilateral crossbite is to use a removable appliance incorporated with finger springs. Alternatively, a removable appliance with a jackscrew, sectioned asymmetrically, can be used. An alternative treatment is to use fixed lingual maxillary expansion appliances. W-arches and Quad-helix appliances can be modified by changing the length of the arms to include more teeth in the anchorage unit. This requires less overall treatment time and cost effective compared to removable appliances ${ }^{(9)}$.

\section{Mandible appliance:}

Mandibular Symphyseal Distraction Osteogenesis(MSDO) - In this an osteotomy is performed after which the healing callus is gradually stretched' Ilizarov's principles are applied including the need for a latency period, rhythm, consolidation period and the rigidity of the distractor during distraction. Lip bumpers, Schwartz devices or functional devices are commonly used to treat transverse mandibular deficiency in growing patients. These method tend to produce only limited dimensional change and the long term stability is questionable. Advantages of DO are greater predictability, more physiologic improvements and better stability. Although it has several advantages, the surgeon must be careful because it could cause periodontal defects or ankylosis of the involved teeth if excess osseous tissue is removed or injuring the periodontal ligament. This also can cause phonetic problems and TMJ disorders ${ }^{(10)}$.

\section{Discussion:}

In Orthodontics, asymmetry is one of the most difficult problems to correct. A functional shift of the mandible in growing patients can be caused by malposed teeth, dental crossbites,or a constricted maxillary arch in growing children. Abnormal tooth contacts causes subsequent mandibular displacement in maximum intercuspation. These can be corrected by aligning teeth, occlusal adjustments and maxillary expansions ${ }^{(12)}$. The children with unilateral posterior crossbite tend to have abnormal chewing patterns and often characterized by reverse sequencing ${ }^{(17)}$. Correction of functional posterior crossbite in the mixed dentition as early as possible after diagnosis has been recommended. If left untreated, can have deleterious effects on the development and functions of the TMJ's ${ }^{(14)}$. It occurs more frequently when chewing on the crossbite side and on harder foods.

\section{Conclusion:}


It should be emphasized that, it is very important to correct at an early age, during the first stage of mixed dentition, where these appliances (rapid maxillary expanders) can be used to open the midpalatal suture and correct transverse skeletal problems. This can be performed in growing patients where the suture expands on treatment, whereas in adult patients, only dentoalveolar expansion is possible ${ }^{(8)}$. It is also understood from the review that early correction of unilateral posterior crossbite give rise to stable result. Expansion appliances should be an integral part of practitioner's armamentarium. However, an appropriate diagnosis, problem list and integrated long time treatment plan should first be developed ${ }^{(7)}$.

\section{References:}

[1]. Sofia Petren, DDS; Lars Bondemark, DDS, Odont DR; Bjorn Soderfeldt, PhD, Dr Med Sc. A Systematic Review Concerning Early Orthodontic Treatment Of Unilateral Posterior Crossbite. Angle Orthod 2003; 73:588-596.

[2]. David B. Kennedy, Matthew Osepchook. Unilateral Posterior Crossbite With Mandibular Shift: A Review. J Can Dent Assoc 2005; 71(8):569-73.

[3]. Chris van Keulen, Guy Martens and Luc Dermaut .Unilateral posterior crossbite and chin deviation: is there a correlation? European Journal Of Orthodontics 26 (2004) 283-288.

[4]. Proffit et al, Posterior crossbites

[5]. Stasa Melink, Mojca Velikonja Vagner, Irena Hocevar-Boltezar, and Maja Ovsenik. Posterior crossbite in the deciduous dentition period, its relation with sucking habits, irregular orofacial functions, and otolaryngological findings. Am J Orthod Dentofacial Orthop 2010; 138:32- 40.

[6]. Maria Grazia Piancino, Francesca Talpone, Paola Dalmasso, Cesare Debernardi, Arthur Lewin and Pietro Bracco. Reversesequencing chewing patterns before and after treatment of children with a unilateral posterior crossbite. European Journal of Orthodontics 28 (2006) 480-484.

[7]. Robert E. Binder, DMD. Correction of Posterior Crossbites: Diagnosis and Treatment. Pediatr Dent.2004; $26: 266-272$.

[8]. Castaner-Peiro A. Interceptive Orthodontics: The need for early diagnosis and treatment of posterior crossbites. Med Oral Patol Oral Cir Bucal 2006;11: E210-4.

[9]. M. Serdar Toroglu, DDS, PhD, Erhan Uzel, DDS, Mustafa Kayalioglu, DDS, and Ilter Uzel, DDS, PhD. Asymmetric maxillary expansion(AMEX) appliance for treatment of true unilateral posterior crossbite. Am J Orthod Dentofacial Orthop 2002; 122: 16473 .

[10]. Nurhat Ozkalayci, DDS, PhD, Mete Ozer, Mahmut Sumer. Treatment of unilateral buccal crossbite with mandibular symphyseal distraction osteogenesis. Korean J Orthod 2011; 41 (1):59-69.

[11]. Silvio Rosan de Oliveira. Dental Press J Orthod 2010 Sept-Oct; 15(5):182-91.

[12]. Kiyoshi Tai, Jae Hyun Park, Kazuhisa Ikeda, et al. Am J Orthod Dentofacial Orthop 2012; 142:509-23.

[13]. Alamoudi N. Thew correlation between occlusal characteristics and temperomandibular dysfunction in Saudi Arabian children. J Clin Pediatr Dent 2000; 24 (3):229-36.

[14]. Defne Kecik, Ilken Kocardereli, and Isil Saatci. Am J Orthod Dentofacial Orthop 2007; 131:202-15.

[15]. Hesse KL, Artun J, Jondeph DR, Kennedy DB. Changes in condylar position and occlusion associated with maxillary expansion for correction of functional unilateral posterior crossbite. Am J Orthod Dentofacial Orthop 1997; 111(4): 410-8.

[16]. Paul Henrik Nerder*, Merete Bakke** and Beni Solow*.European Journal of Orthodontics 21 (1999) $155-166$.

[17]. Gaylord S.Throckmorton, PhD, Peter H.Buschang,MA,PhD, Haruaki Hayasaki,DDS,PhD , and Ary Santos Pinto, DDS ,PhD ${ }^{\mathrm{a}}$. Am J Orthod Dentofacial Orthop 2001; 120:521-9. 